PAEDIATRIC LUNG DISEASE

\title{
Risk factors for the development of bronchiolitis obliterans in children with bronchiolitis
}

\author{
A J Colom, A M Teper, W M Vollmer, G B Diette
}

See end of article for authors' affiliations

.....................

Correspondence to: Dr A J Colom, España 880, San Miguel (1663) Buenos Aires, Argentina; Acolor@intramed.net

Received 2 May 2005 Accepted

15 February 2006

Published Online First

3 March 2006

\begin{abstract}
Background: Bronchiolitis obliterans $(\mathrm{BO})$ is an uncommon and severe form of chronic obstructive lung disease in children that results from an insult to the lower respiratory tract.

Methods: A case-control study of children under the age of 3 years was performed in 109 cases and 99 controls to determine risk factors for the development of BO. Participants were evaluated by immunofluorescence viral tests, pulmonary function tests, and questions to assess tobacco and other exposures.

Results: Bronchiolitis due to adenovirus (odds ratio (OR) 49,95\% confidence interval (CI) 12 to 199) and the need for mechanical ventilation (OR $11,95 \% \mathrm{Cl} 2.6$ to 45 ) were strongly and independently associated with an increased risk for $\mathrm{BO}$. Factors not associated with post-infectious $\mathrm{BO}$ included age of the child, sex, and environmental tobacco exposure (either in utero or during infancy).

Conclusions: Adenovirus infection and need for mechanical ventilation are significant risk factors for developing $\mathrm{BO}$ in children. Further research is needed to determine why these risk factors are so strong and how they may contribute to the development of the disease.
\end{abstract}

B ronchiolitis obliterans (BO) is an uncommon and severe form of chronic obstructive lung disease in children that results from an insult to the lower respiratory tract, which occurs in a number of forms. Included in the clinical scenarios reported in the literature are after Stevens-Johnson syndrome, as a complication of graft versus host disease in bone marrow transplant recipients, and as a manifestation of chronic graft rejection in lung transplant recipients. However, in many parts of the world BO is most commonly seen in children after severe viral lower respiratory tract infections. ${ }^{1}$ Clinically, it is characterised by tachypnoea, increased anteroposterior chest diameter, crackles, wheezing, and hypoxaemia for at least 30 days after the initial injury. ${ }^{2}$ Pathologically, BO is characterised by luminal obstruction with inflammation, granulation tissue, and/or fibrosis and obliteration of the small airways and bronchiectasis. ${ }^{3}$

Post-infectious $\mathrm{BO}$ has been reported mainly in the southern hemisphere (Argentina, Chile, southern Brazil, Australia, and New Zealand) where its impact can be profound. In the respiratory centre at Ricardo Gutiérrez Children's Hospital in Buenos Aires, for example, postinfectious BO accounted for $14 \%$ of all bed-days during the 10 year period from 1993 to 2002.

A number of respiratory viruses-including respiratory syncytial virus (RSV), ${ }^{4}$ parainfluenza, ${ }^{5}$ influenza, ${ }^{6}$ and especially adenovirus $(\mathrm{AV})^{7}$-were associated with severe lung injury leading to BO. Although BO has been observed commonly after viral infections, research has not yet quantified the importance of specific viruses as risk factors for BO. Our current knowledge of post-infectious BO is based largely on a few case series in which the antecedent risk factors have not been clearly identified. ${ }^{8-10}$

To address this knowledge gap, we performed a casecontrol study of children in Buenos Aires, Argentina to determine risk factors for the development of post-infectious $\mathrm{BO}$.

\section{METHODS}

Potential cases included all children treated for postinfectious BO from 1991 to 2002 at the Children's Hospital in Buenos Aires, Argentina (identified from retrospective chart review). The children were aged 3 years or younger with chronic respiratory insufficiency (defined as tachypnoea, increased anteroposterior chest diameter, crackles, wheezing, and hypoxaemia $\left(\mathrm{SaO}_{2}<93 \%\right)$ lasting for at least 30 days after the initial injury) following severe bronchiolitis in a previously healthy infant. ${ }^{2}$ Children with co-existing cystic fibrosis, congenital heart disease, or primary ciliary dyskinesia were excluded. One hundred and nine children met these criteria

Control subjects were identified from the population of new cases of bronchiolitis (first episode of wheeze) who were admitted to our hospital during 2002 and who did not subsequently develop BO. A 12 month period was chosen to encompass the full spectrum of bronchiolitis cases seen during a year and thus to avoid biases associated with seasonality. Similar exclusion criteria were applied to controls as to cases. A total of 161 potential controls were identified in this manner, 99 of whom met the inclusion/exclusion criteria.

Indirect inmunofluorescence viral testing of nasopharyngeal secretions was performed to identify AV, RSV, influenza, and parainfluenza virus. These tests are routinely done on patients admitted for bronchiolitis so were available in the medical records of most cases (64\%) and controls (93\%). Several patients with post-infectious BO were initially treated in other hospitals and viral test results were not available for them.

Chest radiographs and HRCT scans were reviewed and interpreted by two experienced paediatric radiologists. Pulmonary function tests (PFTs) were routinely performed only in patients with BO. They were carried out when the patient's condition was stable (absence of an acute respiratory tract infection for 30 days, lack of upper airway secretions, and normal haemodynamic parameters). Partial forced flow/volume curves and compliance and resistance

Abbreviations: AV, adenovirus; $\mathrm{BO}$, bronchiolitis obliterans; $\mathrm{ETS}$ environmental tobacco smoke; PFT, pulmonary function test; RSV respiratory syncytial virus; $V^{\prime}$ maxFRC, maximal flow at functional residual capacity 


\begin{tabular}{|c|c|c|c|c|c|}
\hline & \multicolumn{2}{|c|}{ Progressed to $B O$} & \multirow[b]{2}{*}{ OR } & \multirow[b]{2}{*}{$95 \% \mathrm{Cl}$} & \multirow[b]{2}{*}{$p$ value } \\
\hline & Yes $(n=109)$ & No $(n=99)$ & & & \\
\hline Median (range) age (months) & $6(1-26)$ & $5(1-20)$ & & & \\
\hline$<6$ months & $67 \%$ & $72 \%$ & 0.5 & 0.3 to 0.9 & 0.02 \\
\hline Male & $60 \%$ & $58 \%$ & 1.1 & 0.6 to 2 & 0.8 \\
\hline Admitted in fall or winter & $75 \%$ & $98 \%$ & 1.0 & 0.8 to 1.2 & 0.9 \\
\hline ETS exposure in uterus & $24 \%$ & $12 \%$ & 1.6 & 0.7 to 4 & 0.3 \\
\hline Current ETS exposure & $59 \%$ & $38 \%$ & 2.2 & 1.2 to 4 & 0.01 \\
\hline Adenovirus infection & $72 \%$ & $3 \%$ & 83 & 22 to 441 & $<0.001$ \\
\hline Mechanical ventilation & $34 \%$ & $3 \%$ & 12 & 5 to 34 & $<0.001$ \\
\hline
\end{tabular}

measurements were done according to the ATS/ERS guidelines. ${ }^{11}$ Values of maximal flow at functional residual capacity (V'maxFRC) were expressed as Z scores. ${ }^{12}{ }^{13}$

Data on current exposure to environmental tobacco smoke (ETS) and exposure to ETS during pregnancy were obtained from the medical records or from interviews with the parents.

\section{Statistical analysis}

Assuming that $10 \%$ of the control subjects would have had exposure to AV, the study had $80 \%$ power to detect a $16 \%$ difference in AV exposure probabilities between cases and controls. Risk factors were assessed using logistic regression analysis and the results are reported as odds ratios (OR) with 95\% confidence intervals (CI). Interaction terms were used to test for lack of additivity of effects on the $\ln$ (odds) scale. The following variables were considered as potential risk factors for post-infectious BO: sex, prenatal and postnatal ETS exposure, age $<6$ months at time of admission for bronchiolitis, evidence of specific viral aetiologies for the bronchiolitis, and the need for mechanical ventilation during the target admission. The 6 month age cut off was used because $67 \%$ of the patients were younger than 6 months.

Analyses were performed using Stata for Windows (Stata Corp, College Station, TX, USA) and Epi-Info (US Department of Health and Human Services/Centers for Disease Control and Prevention, Atlanta, USA) statistical software packages. A p value of $\leqslant 0.05$ indicated statistical significance.

\section{RESULTS}

Participants ranged in age from 1 to 26 months at the time of their initial admission for bronchiolitis, with a mean age of 7 months among cases and 5 months among controls

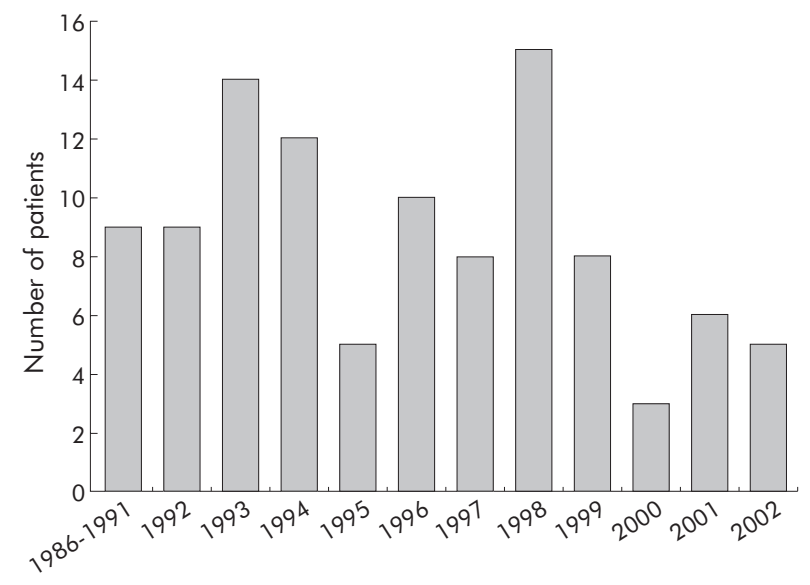

Figure 1 Cases of post-infectious BO by calendar year. (table 1); $60 \%$ of cases and $58 \%$ of controls were boys. The number of new cases of post-infectious BO during the period of the study showed substantial variation from year to year (fig 1). Almost all (98\%) of the controls were admitted in the fall or winter compared with $75 \%$ of patients with postinfectious BO. In those post-infectious BO patients tested, AV was detected in $72 \%$, RSV in $10 \%$, parainfluenza in $4 \%$, and influenza in $1 \%$. In control patients, RSV was the virus most frequently identified $(58 \%)$, and only $3 \%$ were positive for AV. $34 \%$ of patients with post-infectious BO required mechanical ventilation compared with only $3 \%$ of patients in the control group. The median stay in hospital was 30 days (range 11-120) in post-infectious BO patients and 6 days (range 1-40) in controls.

In bivariate analyses AV infection (OR 83, 95\% CI 22 to 441 ) and the need for mechanical ventilation (OR 12, 95\% CI 5 to 34 ) were exceptionally strong risk factors (table 1), and persisted as significant predictors of risk for post-infectious $\mathrm{BO}$ in multivariate logistic regression models (table 2). A formal test for interaction in the logistic model was not significant.

\section{Clinical findings}

Chest radiography and PFTs were performed at the time of the clinical evaluation of the post-infectious $\mathrm{BO}$ cases, which occurred a mean of 4 months after the onset of illness (range 5-30). Patients with $\mathrm{BO}$ had high respiratory rates (mean 37 breaths/min, range 35-40); 70\% (56/80) had a rigid thorax, 74\% (69/93) had wheeze, and 89\% (81/91) had persistent productive cough. Oxygen saturation was lower than normal (mean (SD) $\mathrm{SaO}_{2} 92(5) \%$ ). Most children were not malnourished (mean (SD) Z score length for age -1.6 (1), weight for length -0.45 (1)).

Chest radiographs from 98 of the patients with postinfectious BO showed air trapping (96\%), atelectasis (75\%), areas with an increased interstitial pattern (55\%), and honeycombing $(34 \%)$. The most frequent findings on the HRCT scans (reported for 62 of the cases) were mosaic perfusion $(60 \%)$, bronchiectasis $(58 \%)$, and atelectasis $(44 \%)$.

Table 2 Multivariate logistic regression analysis of risk factors for $\mathrm{BO}$

\begin{tabular}{llll}
\hline Variable & OR & $95 \% \mathrm{Cl}$ & p value \\
\hline Age (<6 $v \geqslant 6$ months) & 1.4 & 0.4 to 5.4 & 0.6 \\
Sex (male $v$ female) & 0.8 & 0.2 to 2.6 & 0.7 \\
ETS at present & 1.4 & 0.4 to 4.5 & 0.5 \\
ETS during pregnancy & 0.4 & 0.1 to 3.2 & 0.4 \\
Adenovirus infection & 49 & 12 to 199 & $<0.001$ \\
Mechanical ventilation & 11 & 2.6 to 45 & 0.001 \\
\hline \multirow{2}{*}{ ETS, environmental tobacco smoke. }
\end{tabular}




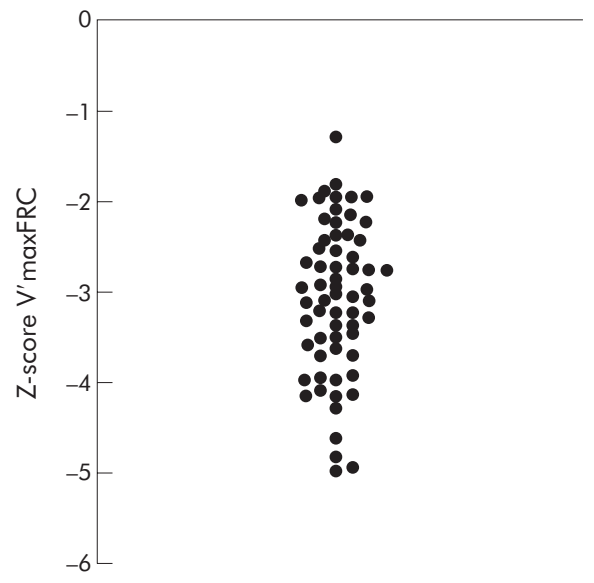

Figure 2 Pulmonary function of patients with post-infectious BO.

Pulmonary function tests were performed in 75 of the 109 patients with post-infectious BO (68\%) and showed severe fixed airflow obstruction (mean (SD) V'maxFRC Z score -3.1 (0.8); fig 2), decreased compliance (1.21 (0.5) ml/ $\mathrm{cm} \mathrm{H}_{2} \mathrm{O} / \mathrm{kg}$ ), and increased resistance $\left(0.05(0.02) \mathrm{cm} \mathrm{H}_{2} \mathrm{O}\right.$ / $\mathrm{ml} / \mathrm{seg}$ ). The mean bronchodilator response was $7 \%$. Some patients did not perform the test because they were more than 2 years old or because of the severity of the illness.

Most of the patients with post-infectious BO (57\%) required oxygen supplementation after discharge from the initial hospital admission for a median period of 17 months (range 1-62), and the majority (95\%) required readmission to hospital for subsequent infections of the lower respiratory tract. Hypoxaemia improved slowly over several years and only $3 \%$ (1/37) remained on supplemental oxygen at the age of 6 years.

\section{DISCUSSION}

This study includes the largest sample of paediatric patients with post-infectious $\mathrm{BO}$ in the medical literature to date. The results show that $\mathrm{AV}$ infection and the need for mechanical ventilation are strong independent risk factors for developing post-infectious BO in children under 3 years of age. During the last two decades there have been a growing number of reports of patients with post-infectious BO. Although there are no worldwide studies of the prevalence of post-infectious $\mathrm{BO}$, it has mainly been reported in certain areas including Argentina, Brazil, Chile, New Zealand, Canada, and South Korea. For unknown reasons, large areas such as the US and Europe have had only a small number of reports. ${ }^{2-10}$ Reasons for the difference in the prevalence of the disease may include the severity of the AV infections according to the serotype and to a genetic predisposition to develop the disease, particularly among unique populations derived from populations native to those regions. ${ }^{14-17}$

The central role of AV in the development of postinfectious $\mathrm{BO}$ has been well documented. ${ }^{14}$ In our study AV was identified in $71 \%$ of the patients with post-infectious BO in whom diagnostic testing was performed. Although since 1984 a new genotype of adenovirus, AV7h, has stood out as the most virulent serotype, other AVs (such as 3, 5, and 21) also cause $\mathrm{BO} .^{15}$ Epidemiological studies indicate that the global prevalence patterns of AV genome types do shift over time and geographical region, which may make it technically difficult to develop a new vaccine with broad applicability. ${ }^{18}$

Patients with severe AV infection have been shown to have immune complexes containing AV antigen in the lung, as well as increased serum levels of interleukin-6, interleukin-8, and tumour necrosis factor- $\alpha .^{19}{ }^{20}$ These previous studies suggest that a specific immunological response may be important in the development of BO following AV infection. ${ }^{21}$ In the present study, illness occurred in very young infants$67 \%$ were younger than 6 months and 93\% were younger than 12 months-but our findings did not show that age was a risk factor for developing post-infectious BO. In previous studies patients admitted with bronchiolitis and malnutrition had a more severe course; ${ }^{22}$ however, malnutrition was not an important factor in the present study as most of the children were not malnourished.

Although AV was identified in most of the post-infectious BO patients at the onset of the illness, other viruses were also found. RSV is the most frequent casual agent of bronchiolitis and occurs mainly during winter months. An association between BO and RSV has been reported only rarely, ${ }^{43}$ and the pathogenesis of $\mathrm{BO}$ is not clear in these patients. Simultaneous infections of RSV and AV have also been reported. ${ }^{23}{ }^{24}$ In these cases it would seem most likely that the $\mathrm{AV}$ infection is the cause of BO. About one quarter of the total diagnosed cases infected with influenza develop complications, mostly in younger children (0-4 years of age). ${ }^{25}$ However, $\mathrm{BO}$ is a rare complication of influenza with only a few cases reported in infancy and early childhood. ${ }^{6}$

The use of mechanical ventilation, an important treatment for children in intensive care units, has apparently allowed severely ill children to survive who previously would have died before BO could be recognised. Mechanical ventilation is indispensable for the support of critically ill patients with respiratory insufficiency. Although our study found that mechanical ventilation was a significant risk factor for post-infectious $\mathrm{BO}$, our results do not indicate whether it causes injury to the lung that increases the risk for developing post-infectious BO or whether it merely serves as an indicator of severity of illness. Mechanisms by which mechanical ventilation could cause lung injury include untoward effects from volutrauma, oxygen toxic effects, and barotrauma. ${ }^{26}$ Further research is needed to clarify the relationship between mechanical ventilation and postinfectious $\mathrm{BO}$, and to study whether lung protective strategies are needed for this vulnerable population. Cidofovir, a new antiviral agent is under investigation and may be an alternative for the treatment of AV infection in the future. ${ }^{27}$

The ethnic background of patients is another factor that has been linked with the risk for developing post-infectious BO. Two ethnic indigenous populations of children in New Zealand and central Canada ${ }^{16}{ }^{17}$ have been reported to be particularly susceptible to developing post-infectious $\mathrm{BO}$, which suggests that genetic factors may be important. ${ }^{28}$ Our study evaluated children of different geographical origins but did not assess ethnicity. The relationship of post-infectious $\mathrm{BO}$ to race/ethnicity is an interesting and important topic for future research.

All tested patients with post-infectious BO had a V'maxFRC that was more severely affected than in other diseases such as bronchopulmonary dysplasia or asthma which, even in their most severe forms, usually respond to bronchodilators. This finding confirms our previous report about the use of PFTs which enable us to improve the diagnostic approach to post-infectious $\mathrm{BO} .{ }^{2}$ We believe that the patient's clinical history and the radiological and HRCT images are sufficient in most cases to confirm the diagnosis and to differentiate post-infectious BO from other pulmonary disorders. ${ }^{2}$ These clinical evaluations should be considered in tandem with the functional pattern which, in post-infectious $\mathrm{BO}$, is characterised by severe and fixed obstruction, an increase in resistance, and a decrease in lung compliance. In the few cases in which doubt persists about the diagnosis, a lung biopsy may be needed. 
We conclude that both AV infection and mechanical ventilation are strongly associated with the development of post-infectious BO. The immunological response of the host and genetically determined factors could be additional factors that modify or increase susceptibility to the condition. Strategies to prevent infection with AV, such as development of a vaccine, should be encouraged to prevent this devastating illness. In the meantime, early recognition is vital so that children can be isolated to prevent the spread of infection to others. When AV is suspected or isolated, early aggressive treatment is warranted, and future clinical studies are required to determine the best treatment strategies.

\section{ACKNOWLEDGEMENTS}

The authors thank Dr Sonia Buist and the staff of the ATS International Respiratory Epidemiology Course for encouraging our research and helping to improve the paper, and Dr Carlos Kofman for performing most of the infant pulmonary function tests.

\section{Authors' affiliations}

A J Colom, A M Teper, Respiratory Center, R. Gutiérrez Children's Hospital, Buenos Aires, Argentina

W M Vollmer, Kaiser Permanente Center for Health Research, Portland, Oregon, USA

G B Diette, Division of Pulmonary and Critical Care Medicine, Johns Hopkins University School of Medicine, Baltimore, Maryland, USA

Funding: Methods in Epidemiologic, Clinical and Operations Research (MECOR program), American Thoracic Society.

Competing interests: none.

\section{REFERENCES}

1 Hardy KA, Schidlow DV, Zaeri N. Obliterative bronchiolitis in children. Chest 1988;93:460-6.

2 Teper AM, Kofman CD, Maffey AF, et al. Lung function in infants with chronic pulmonary disease after severe adenoviral illness. J Pediatr 1999;134:730-3.

3 Becroft DMO. Bronchiolitis obliterans, bronchiectasis, and other sequelae of adenovirus type 21 infection in young children. J Clin Pathol 1971;24:72-82.

4 Krasinski K. Severe respiratory syncytial virus infection: clinical features, nosocomial acquisition and outcome. Pediatr Infect Dis J 1985;4:250-7.

5 Welliver R, Wong D, Sun $M$, et al. Parainfluenza virus bronchiolitis. Am J Dis Child 1986;140:34-40.

6 Laraya-Cuasay LR, DeForest A, Huff D, et al. Chronic pulmonary

complications of early influenza virus infection in children. Am Rev Respir Dis 1977;116:617-25.
7 Simila $S$, Linna $O$, Lanning $P$, et al. Chronic lung damage caused by adenovirus type 7: a ten year follow up study. Chest 1981;80:127-31.

8 Chang A, Masel J, Masters B. Post-infectious bronchiolitis obliterans: clinical, radiological and pulmonary function sequelae. Pediatr Radiol 1998;28:23-9.

9 Zhang L, Irion K, Kozakewich H, et al. Clinical course of postinfectious bronchiolitis obliterans. Pediatr Pulmonol 2000;29:341-50.

$10 \mathrm{Kim}$ CK, Kim SW, Kim JS, et al. Bronchiolitis obliterans in the 1990s in Korea and the United States. Chest 2001;120:1101-6.

11 Hoo AF, Dezateux C, Hanrahan JP, et al. Sex-specific prediction equations for $V_{\max }(\mathrm{FRC})$ in infancy: a multicenter collaborative study. Am J Respir Crit Care Med 2002; 165:1084-92.

12 Hanrahan JP, Tager IB, Castile RG, et al. Pulmonary function measures in healthy infants. Variability and size correction. Am Rev Respir Dis 1990;141:1127-35.

13 Hiatt $\mathbf{P}$, Eigen $\mathrm{H}, \mathrm{Yu}$, et al. Bronchodilator responsiveness in infants and young children with cystic fibrosis. Am Rev Respir Dis 1988;137:119-22.

14 Sly PD, Soto-Quiros ME, Landau LI, et al. Factors predisposing to abnormal pulmonary function after adenovirus type 7 pneumonia. Arch Dis Child 1984:59:935-9.

15 Kajon A, Mistchenko A, Videla C, et al. Molecular epidemiology of adenoviruses associated with acute lower respiratory infections of children in the south cone of South America (1991-1994). J Med Virol 1996;48:151-6.

16 Lang W, Howden C, Laws J, et al. Bronchopneumonia with serious sequelae in children with evidence of adenovirus type 21 infection. BMJ 1969;1:73-9.

17 Wenman W, Pagtakhan R, Reed M, et al. Adenovirus bronchiolitis in Manitoba. Epidemiologic, clinical and radiologic features. Chest 1982;81:605-9.

18 Purkayastha A, Su J, McGraw J, et al. Genomic and bioinformatics analyses of HAdV-4vac and HAdV-7vac, two human adenovirus (HAdV) strains that constituted original prophylaxis against HAdV-related acute respiratory disease, a reemerging epidemic disease. J Clin Microbiol 2005;43:3083-94.

19 Mistchenko AS, Lenzi HL, Thompson FM, et al. Participation of immune complexes in adenovirus infection. Acta Paediatr 1992;81:983-8.

20 Mistchenko AS, Diez RA, Mariani AL, et al. Cytokines in adenoviral disease in children: association of interleukin-6, interleukin-8, and tumor necrosis factor alpha levels with clinical outcome. J Pediatr 1994;124:714-20.

21 Mistchenko AS, Koch ERR, Kajon AE, et al. Lymphocyte subsets and cytokines in adenoviral infection in children. Acta Paediatr 1998;87:933-9.

22 Law BJ, Carbonell-Estrany X, Simoes EA. An update on respiratory syncytial virus epidemiology: a developed country perspective. Respir Med 2002;96(Suppl B):S1-7.

23 Massie R, Armstrong D. Bronchiectasis and bronchiolitis obliterans post respiratory syncytial virus infection: think again. J Paediatr Child Health 1999;35:497-8.

24 Hirschheimer M, Silva $P$, Giudici R, et al. Simultaneous viral infection and childhood bronchiolitis obliterans. Braz J Infect Dis 2002;6:146-8.

25 Loughlin J, Poulios N, Napalkov P, et al. A study of influenza and influenzarelated complications among children in a large US health insurance plan database. Pharmacoeconomics 2003;21:273-83.

26 Pinhu L, Whitehead T, Evans T, et al. Ventilator-associated lung injury. Lancet 2003;361:332-40.

27 De Clercq $\mathrm{E}$. Clinical potential of the acyclic nucleoside phosphonates cidofovir, adefovir, and tenofovir in treatment of DNA virus and retrovirus infections. Clin Microbiol Rev 2003;16:569-96.

28 Wohl ME, Chernick V. State of the art: bronchiolitis. Am Rev Respir Dis 1978;118:759-81. 